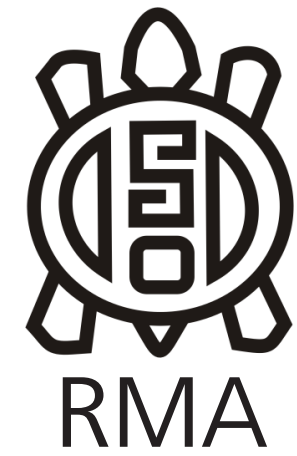

Arqueología

\section{Improntas y cerámica en ensamble. La producción de cestas en tiempos precoloniales en las sociedades agroalfareras de la región de Villa de Soto, Córdoba}

Imprints and ceramic in assembly. The production of baskets in precolonial times in the agro-pottery societies of the Villa de Soto region, Córdoba

Marcos Abalos Luna*

*Instituto de Antropología de Córdoba, CONICET, Museo de Antropología, Facultad de Filosofía y Humanidades, Universidad Nacional de Córdoba, Argentina. E-mail: marcos.abalosluna@gmail.com

\begin{abstract}
Resumen
La fabricación de cestas fue una práctica extendida para las sociedades agroalfareras que poblaron la región de Villa de Soto, Córdoba, en tiempos inmediatamente previos a la implantación de la corona española. Las condiciones ambientales de esta zona no hacen posible la conservación de material orgánico, sin embargo, las improntas en las superficies de las vasijas constituyen una evidencia indirecta que, con los recaudos necesarios, se transforman en una importante fuente de información sobre las técnicas cesteras. Se presenta aquí el análisis de la producción cestera a partir de fragmentos cerámicos con improntas de cestería provenientes de dicha región. Con la utilización de un código analítico descriptivo y la aplicación de técnicas estadísticas de análisis multivariado (cluster analysis), arribamos a dos técnicas características en la confección de las cestas, espiralado simple (coiled) y encordado envuelto (wrapped twining), pudiendo distinguir variedades dentro de las mismas. Estos resultados nos permiten desentrañar algunos de los diferentes eventos del tejer en estas sociedades y las múltiples articulaciones que estos conllevan.
\end{abstract}

Palabras clave: Cestería; Técnicas; Estructuras textiles; Improntas; Córdoba.

\begin{abstract}
The manufacture of baskets was a widespread practice for the agro-pottery societies that populated the Villa de Soto region, Córdoba, in times immediately prior to the implantation of the Spanish crown. The environmental conditions of this area do not make possible the conservation of organic material, however, the imprints on the surfaces of the vessels constitute indirect evidence that, with the necessary precautions, become an important source of information on basket techniques. We present here the analysis of the basketry production from ceramic fragments with basketry imprints from said region. With the use of a descriptive analytical code and the application of statistical techniques of multivariate analysis (cluster analysis), we arrived at two characteristic techniques in the preparation of baskets, simple spiral (coiled) and wrapped string (wrapped twining), being able to distinguish varieties within them. These results allow us to unravel some of the different events of weaving in these societies and the multiple articulations that these entail.
\end{abstract}

Keywords: Basketry; Techniques; Textile structures; Imprints; Cordoba.

La confección de una cesta, al igual que muchas otras actividades tales como la recolección de alimentos, la talla de artefactos, el armado de un fogón, etc., posee un ritmo particular que va emparentado a un devenir construido dentro de un paisaje de tareas. Como Ingold (2008) ha señalado, tejer una cesta es más que una serie de pasos técnicos con miras a un objetivo (la cesta), es un proceso de construcción donde diversos ritmos entran en juego. El objetivo del siguiente artículo es identificar y caracterizar las técnicas implicadas en la construcción de las cestas y las relaciones entre los elementos de las estructuras textiles. Esto lo realizaremos de manera indirecta a través del estudio de las improntas de cestas en fragmentos cerámicos provenientes de la región de Soto (Córdoba, Argentina) ya que las condiciones ambientales de la zona no favorecen la conservación de material orgánico, y no se conocen ejemplares de las cestas utilizadas. Las improntas en las superficies de las vasijas constituyen un registro indirecto que, con los recaudos necesarios, se transforman en una importante fuente de información sobre las técnicas cesteras.

La localidad de Villa de Soto se halla en el Departamento de Cruz del Eje en el NE de la Pcia. de Córdoba, 
Argentina (Figura 1). Allí se han localizado diversos sitios en ambas márgenes del río Soto, en una extensión lineal de aproximadamente $10 \mathrm{~km}$ desde el paraje de la toma del río, al Suroeste del poblado actual, hasta aproximadamente $2 \mathrm{~km}$ aguas abajo. Soto fue declarado "pueblo de indios" por parte de la corona española (Tell y Castro Olañeta 2011) y, si bien se lo ha documentado en gran cantidad de fuentes etnohistóricas (Piana 1992, González Navarro 2010), la zona de estudio cuenta con un solo trabajo arqueológico. En 1943 Alberto Rex González publicó "El paradero indígena de Soto" producto de un trabajo de campo realizado varios años atrás. Allí describe y analiza diferentes materiales hallados por él y donados por los vecinos. Entre estos se hallan puntas de proyectil, hachas, raspadores, perforadores, chaquiras, una figura antropomorfa, un collar de caracoles. También menciona la presencia de fragmentos cerámicos con improntas de cestas y/o redes en sus superficies. Actualmente no contamos con cronología absoluta, sin embargo, a partir de los conjuntos artefactuales hallados en prospecciones y en una reciente excavación (que coinciden con los materiales descriptos por Rex González), podemos situar estos grupos agroalfareros en tiempos inmediatamente previos a la implantación de la corona española, en lo que denominamos tiempos precoloniales (Laguens et al. 2017). Si nos referimos específicamente a la cerámica con improntas, encontramos las huellas de las mismas estructuras textiles en los fragmentos de los sitios C. Pun.39 (854 \pm 39 AP) (Medina 2010), y Arroyo Tala Cañada 1 (900 \pm 70 AP) (Pastor 2007).

En esta zona, actualmente desarrollamos un proyecto arqueológico ${ }^{1}$ con miras a la comprensión de los modos de vidas locales partiendo desde "la escala de la vida diaria, de la cotidianeidad de las prácticas materiales, que, en su repetición, acumulación y sucesiva modificación en eventos puntuales, breves, ponen en escena a su vez estructuras, formas de entender el mundo y formas de hacer las cosas de mucha más larga duración" (Laguens et. al 2017:4). Es dentro de este enfoque que abordamos la elaboración de cestas, y otras prácticas, a partir de las múltiples repeticiones entrelazadas que componen ese "hacer". De este modo, cada práctica posee un ritmo particular que se caracteriza por su reproducción con ciertas frecuencias e intermitencias (en lo que puede entenderse como una duración cualificada, un tiempo diferenciado) (Lefebvre 2004). A través de los ritmos, las cosas, los lugares y los cuerpos se van constituyendo y adquiriendo identidad, y son las tareas las que dejan huellas en materia, herramientas y lugares (Mlekuz 2014). Avanzar por estos senderos significa, también, considerar al mundo en constante movimiento, y, sobre todo, en procesos. En esta ocasión daremos un primer paso para tratar de identificar las posibles técnicas cesteras empleadas.

1 Re-ensamblando la arqueología de Chihimi Sei (Valle de Soto, Córdoba): cosas, personas, memorias, tiempos y territorios en relación (Secyt 2018-2019).

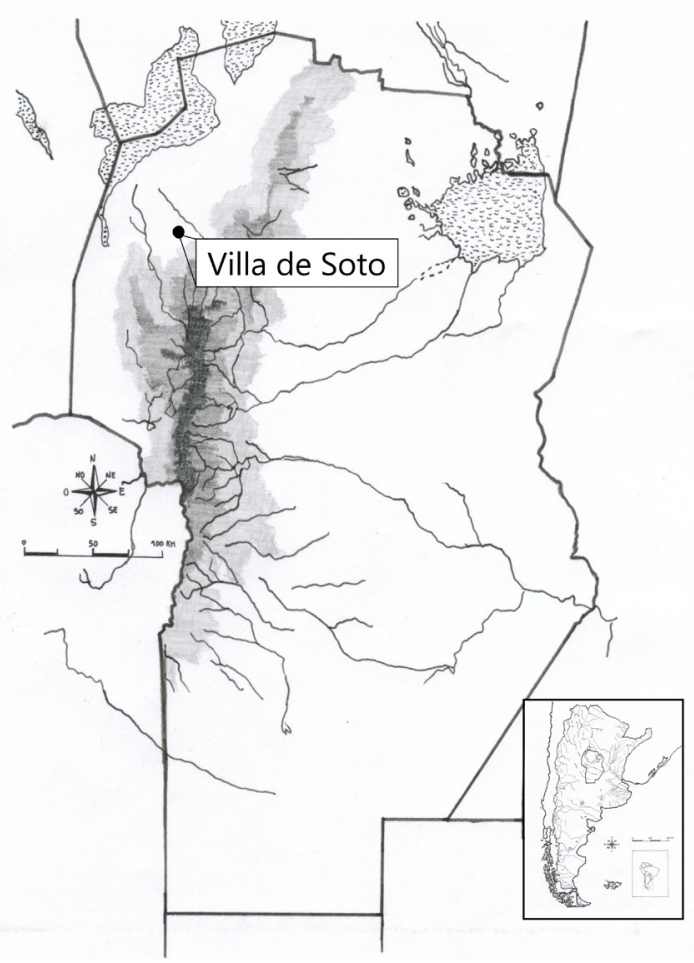

Figura 1. Ubicación de Villa de Soto. Mapa confeccionado por Paola Franco.

Figure 1. Location of Villa de Soto. Map made by Paola Franco.

\section{Antecedentes sobre Improntas}

En América del Sur encontramos evidencia de cestería desde hace 3000 años en Patagonia, en sitios cazadores recolectores (Pérez de Micou, Lopez Campeny y Lessa Costa 2014). Así mismo, fue en el Noroeste de Argentina donde se realizaron mayores investigaciones arqueológicas tanto de la cerámica con impresiones como de algunas cestas (Pérez de Micou y Ancíbor 1994; Pérez de Micou 2000, Pérez de Micou 2001; Tarragó y Renard 2001; Calo 2008: López Campeny 2011).

Para la región de las Sierras Centrales, han sido varios los autores que se han abocado al estudio de las técnicas cesteras dada la cantidad y variedad de fragmentos con improntas en los sitios de esta zona. Uno de los primeros fue Gardner (1919), quien analizó fragmentos con improntas textiles de cestas y redes procedentes de tres sitios del Valle de Punilla (Córdoba). En dicha aproximación vale rescatar el esfuerzo del autor por realizar los moldes y reproducir los nudos de las redes. A partir de estos, definió cinco grupos correspondientes a las improntas de cestería. Además, sugirió la existencia de platillos de cestería sobre los que podrían haber sido confeccionadas aquellas vasijas que presentaban improntas solo en su base. 
Antonio Serrano (1945) analizó diversos fragmentos de "alfarería modelada sobre canastos", entre los cuales se encontraban varios ejemplares provenientes de Villa de Soto. A partir de calcos en plastilina, realizó una detallada descripción de los tipos de técnicas, sus posibles materiales y procedencias, tejiendo relaciones con la cerámica con improntas de Santiago del Estero, los llanos de La Rioja y los Valles Calchaquíes. Serrano reconoció dos tipos de manufacturas diferentes en la confección de cestas para los grupos que poblaron la provincia. Una de ellas es coiled o aduja, equivalente al espiralado simple, y la otra es el falso espiralado, equivalente al encordado envuelto, traducción de wrapped twining (Adovasio 1977). Si bien el autor manifiestó el uso utilitario de las vasijas, no descartó una posible función decorativa de las improntas.

Vale destacar el trabajo realizado por Bonofiglio y Herrera (1979) al analizar 320 fragmentos con improntas provenientes de diversos sitios del departamento de Río Segundo (Córdoba). De los mismos, solo un ejemplar corresponde al cuerpo de una vasija; casi la totalidad de la muestra corresponde a bases. A partir de moldes en plastilina, las autoras identificaron las técnicas cesteras y coincidieron con las definidas por Serrano. En esta muestra solo dos ejemplares corresponden al llamado falso espiralado. Las impresiones correspondientes a la técnica aduja solo se encontraron en la base de los fragmentos cerámicos. "Aparentemente se trata de platillos de cesta, utilizados como base para armar sobre ellos la vasija, o para ponerlas a secar" (Bonofiglio y Herrera 1979:1).

Por otro lado, Laguens y Fabra (1999) realizaron un minucioso análisis de 199 fragmentos cerámicos con improntas provenientes de diversos puntos de la provincia de Córdoba a partir del cual identificaron dos técnicas, el espiralado simple (coiled) y el encordado envuelto (wrapped twining). Los autores caracterizaron tres grandes grupos y diferentes variedades en función de la interacción entre la trama y la urdimbre.

Por su parte, Figueroa, Dantas y Pautassi (2011) analizaron las técnicas textiles de fragmentos procedentes del Valle de Punilla (sitios Huaycondo, San Roque, El Fantasio y La Quinta). Como resultado, caracterizaron, al igual que trabajos anteriores, las dos técnicas mencionadas: espiralado simple (coiled) y encordado envuelto (wrapped twining). Además de complementar esto con análisis funcionales de los recipientes con improntas, los autores realizaron comparaciones a nivel regional entre las técnicas. Notaron una marcada utilización del espiralado simple por sobre el encordado envuelto para el Valle de Salsacate (Córdoba) en contraposición a los fragmentos provenientes del Valle de Punilla, donde prevalece el encordado envuelto (Dantas y Figueroa 2008).

Actualmente, Laguens (2017) encara el estudio de estos fragmentos cerámicos ya no como improntas, sino desde la perspectiva del uso de éstas como moldes para su fabricación. Como señala el autor, podemos encontrar, en las Sierras Centrales, improntas de cestería en las bases de piezas cerámicas, lo cual sugiere la utilización de un elemento plano de cesta de apoyo durante el proceso de manufactura; o bien en el cuerpo de las vasijas, para cuya realización sin duda fueron utilizadas las cestas como forma de sustento para el moldeado de las piezas cerámicas a partir de la cobertura de su interior con arcilla. De otra manera, señala el autor, las piezas cerámicas no podrían haber copiado con tanto detalle la morfología de los cestos, ni su forma. Como resultado de ello hoy podemos identificar a las improntas de cestería en la superficie externa de estas piezas cerámicas. Además, Laguens describe una vasija confeccionada con molde de cesta, en donde se observa, a partir de la impronta, la forma abierta y subglobular de la cesta que sirvió de molde. En este caso, la cesta fue utilizada para la manufactura de la mitad inferior de la pieza, mientras que el resto se presenta liso, confeccionado probablemente mediante la otra técnica habitual de rodete. En nuestra muestra encontramos varios fragmentos de puntos angulares de las vasijas en donde se observan estas características.

Fuera de la provincia de Córdoba, cobra especial relevancia para este trabajo el estudio realizado por López Campeny (2011) en Santiago del Estero. La autora identifica varias estructuras textiles y plantea las posibles relaciones con otras áreas del NOA, especialmente para el caso del encordado envuelto cuyo desarrollo se encuentra vinculado al centro y noroeste de Córdoba, Santiago del Estero, La Rioja, Tucumán y Jujuy. Además, plantea los límites y las potencialidades metodológicas a la hora de analizar los fragmentos con improntas, y expone el concepto de técnicas superpuestas (producción cerámica y textil).

\section{Materiales y Métodos}

La muestra analizada se compone de 70 fragmentos cerámicos provenientes de la Colección Serrano que fueron recolectados superficialmente de la margen izquierda del río de Villa de Soto en la excursión realizada por Antonio Serrano, Juan Bialet y Manuel Oliva en 1944. Esta colección pertenece a la Reserva Patrimonial y Archivo del Museo de Antropología de la Facultad de Filosofía y Humanidades de la Universidad Nacional de Córdoba. A cada fragmento del conjunto se lo limpió en seco con cepillo y se le asignó un número correlativo para favorecer la manipulación.

Al igual que las investigaciones previas que han abordado técnicas cesteras a partir de la cerámica, nuestro análisis es indirecto y parte de los fragmentos cerámicos en cuya superficie quedó registro de dichas estructuras textiles. La mayoría de los trabajos que mencionamos en los 
antecedentes han realizado diversos tipos de moldes para pasar del negativo, presente en los tiestos, al positivo $y$, de este modo, obtener, "un plus de información significativo en relación con aquellas situaciones en las que sólo se realiza el estudio directo de los negativos." (López Campeny 223: 2011). Sin embargo, la autora nos advierte sobre la existencia de ciertos problemas con la remanencia de material moderno en los tiestos, dificultando incluso futuras investigaciones que realicen estudios físicoquímicos sobre los mismos. Frente a esto, López Campeny sugiere el uso prudencial de la técnica de moldeado a fin de minimizar el impacto en las muestras. En nuestro caso de estudio, optamos por realizar un número moderado de moldeados, para lo cual seleccionamos fragmentos en función de su estado de conservación y representatividad. Realizamos 10 moldes de masilla de base epóxica por su consistencia, poca lubricación y exactitud en la reproducción de las improntas. El resto de los 60 fragmentos fue analizado a partir del negativo. Vale aclarar que en todas estas etapas se trabajó con lupa binocular con diferentes aumentos, tanto para los moldes de las improntas como para los fragmentos cerámicos, a fin de distinguir las huellas dejadas por los elementos de las estructuras textiles exhibidas en las improntas.

Se utilizó un código analítico descriptivo (Lahitte y Cascardi 1980) para el estudio del material. En una primera etapa, se realizaron dos fichas para la descripción de los fragmentos: una de carácter general (ficha A) y otra para las improntas (ficha B). Los datos arrojados fueron sometidos a análisis estadísticos simples y multivariados, y posteriormente se realizó un análisis cualitativo de los resultados.

La ficha A tuvo como objetivo realizar una descripción general del soporte cerámico donde se conservaron las improntas teniendo en cuenta las siguientes características: largo, ancho, espesor, porción de la pieza, estado de conservación, tipo de cocción, técnicas de manufactura, huellas de uso, tratamiento de superficie interna, tratamiento de superficie externa, color de superficie interna y color de superficie externa. Para realizar esta descripción se ubicó a los tiestos a partir de la identificación de las huellas de alisamiento de las paredes internas o externas. Luego, se ubicó la superficie donde se encontraba la impronta frente al observador.

Una vez finalizada la ficha $A$, se procedió a realizar el análisis de las improntas (ficha B). Ésta tuvo como objetivo abordar los elementos que constituyen los entramados de las estructuras textiles a partir de la definición de ciertas variables. Este tratamiento de los datos permite establecer interrelaciones entre estas variables, y gracias a la combinación de rasgos podemos arribar a diversos interrogantes: ¿la cantidad de elementos de la trama por cruce de fibra depende de la selección del material utilizado para realizar la cestería? ¿en qué posición se encuentran mayoritariamente las improntas en estos fragmentos cerámicos? ¿la cantidad de elementos de la urdimbre depende de las puntadas de la trama?, entre otras cuestiones. Además, este tipo de análisis permite encontrar mayor variabilidad a partir de las combinaciones de los diferentes atributos.

Para la construcción de nuestro código analítico tuvimos en cuenta los diversos tipos de patrones derivados de la interacción entre elementos activos y pasivos del tejido y los trabajos previos sobre la temática. Utilizamos especialmente el abordaje sugerido por Adovasio (1977) y el trabajo realizado por Fabra y Laguens (1999) en la elaboración de algunas variables. También, para la descripción de las estructuras textiles, utilizamos algunos elementos de la terminología de Emery (1966). Además, tomamos como referencia la descripción y medidas de las improntas que realiza Calo (2008) en su estudio sobre los fragmentos con improntas de cestería espiral del sitio Cardonal (Valle del Cajón, Catamarca) para el análisis de los elementos de las estructuras textiles.

De este modo, priorizamos los siguientes atributos: ubicación de la impronta en relación a la superficie del tiesto, tipo de urdimbre (morfología de la estructura pasiva del entramado), tipo de trama (morfología de la estructura activa del entramado), número de elementos de trama por cruce de línea de urdimbre (cantidad de puntadas que realiza el elemento flexible) y número de elementos de trama horizontal (varillas, hilos), número de elementos de la urdimbre, espaciamiento (definir la apertura entre las puntadas de la trama por $\mathrm{cm}$ ), morfología de los elementos de la estructura textil (identificar los diferentes tipos de elementos constitutivos de trama y urdimbre), orientación de los elementos de la trama y de la urdimbre, y superficie del tiesto cubierto con impronta. Además de estos atributos, se procedió a tomar medidas de las improntas de cada uno de los fragmentos cuyo estado de conservación lo permitió. Se tomaron medidas de los diferentes elementos de las estructuras (ancho de los elementos, largo de las puntadas, largo entre las puntadas). Este análisis cuantitativo de las improntas permite obtener información para realizar futuras hipótesis sobre los materiales utilizados en el hacer una cesta. En todos los fragmentos, se efectuaron tres mediciones para cada una de las variables a fin de realizar un promedio entre ellas. Las medidas fueron tomadas utilizando la cámara digital modelo CMOS $1 / 2$ " Motic 1000 con adaptador para lupa binocular de diferentes aumentos.

Luego de aplicar los criterios de las dos fichas al conjunto en estudio, se procedió a realizar el análisis y, a partir de sus resultados, se agruparon los fragmentos en conjuntos. Para arribar a esto se realizó un análisis multivariado, el cual consistió en un análisis de conglomerados a partir del programa PAST versión 3.25 (Hammer, Harper y Ryan 2001). Este procedimiento 
aglomerativo permite jerarquizar las interrelaciones entre los individuos y los conjuntos. En primera instancia, se agrupan aquellos elementos con mayor nivel de similitud, luego se agregan nuevos elementos a los grupos y, a su vez, se agrupan entre sí de modo que todos quedan dentro del mismo conjunto (Shennan 1992). En la realización de esta etapa se tuvieron en cuenta aquellas variables cualitativas del código analítico específico para las improntas (ficha B).

\section{Análisis y Resultados}

A partir de los análisis pudimos determinar que el $87,14 \%(n=61)$ de los tiestos corresponden al sector del cuerpo de piezas, el 5,72\% ( $n=4)$ a bordes, el $2,86 \%$ $(n=2)$ a fragmentos de bases y el $4,28 \%(n=3)$ restante a fragmentos que no pudieron ser asignados a ningún sector. En casi todos estos casos, las improntas se encontraban en la superficie externa de los fragmentos, salvo en uno donde no se pudo distinguir entre las superficies dado el mal estado de conservación. Del total, 60 fragmentos presentan en toda su superficie la impronta, de los 10 restantes las improntas se encuentran en diferentes proporciones tal como lo muestra el gráfico de la Tabla $n^{\circ} 1$. Estos gráficos presentan los valores arrojados para los diferentes atributos del código analítico de la ficha $B$. Entre los resultados se encuentra la inexistencia de urdimbre, ya que no se visualiza en las improntas. Por otro lado, el $70 \%(n=49)$ de los fragmentos presenta dos tipos de trama diferentes (un elemento vertical y otro horizontal), mientras que el resto de las improntas, $30 \%(n=21)$, posee un solo elemento (vertical), esto coincidiría con los antecedentes para la zona de estudio. En el elemento vertical de las estructuras para ambos tipos de trama, hay una predominancia de la trama simple $(35,71 \%)$, es decir, en cada puntada que teje la trama interviene un elemento de esta, sea hilo o fibra. Si tomamos en cuenta el espaciamiento a partir del elemento vertical y flexible de la trama, el que realiza la puntada, contamos con una gran predominancia de un espaciamiento abierto y regular $(57,14 \%)$, en menor medida cerrado (20\%) y varios fragmentos donde no es posible mensurar por el estado de conservación (20\%), y sólo dos estructuras exhiben un espaciamiento abierto irregular (2,86\%). De toda la colección, el 68,58\% ( $n=48)$ de los fragmentos presentan rastros del contacto con un elemento de morfología circular en las puntadas, mientras que el $15,71 \%(n=11)$ corresponde a improntas de elementos planos. En los fragmentos restantes no se pudo distinguir la morfología de los elementos constitutivos de la estructura $15,71 \%(n=11)$. Precisamente, fueron 11 los fragmentos que no se pudieron clasificar debido a las malas condiciones de preservación.

Por sí solos estos atributos poco nos ayudan para inferir los procesos detrás de la producción cestera. Como bien mencionamos, para interrelacionar las variables utilizamos un análisis de conglomerados (cluster analysis) que se encuentra plasmado en el dendograma de la Figura $n^{\circ} 3$. Para realizar este análisis utilizamos dos combinaciones a fin de comparar los resultados entre ambos: enlace medio con distancia euclídea y el algoritmo de Ward. Un total de nueve variables fueron seleccionadas de la ficha $B$, correspondiente al análisis de improntas (tipo de trama, espaciamiento, morfología de elementos de la trama vertical, morfología de los elementos de la trama horizontal, cantidad de elementos de la trama vertical, cantidad de elementos de la trama horizontal, tipo de tramado vertical, orientación de los elementos de trama vertical, orientación de los elementos de la trama horizontal). Ambos diagramas arrojaron resultados similares, especialmente si realizamos un corte a un nivel de similitud bajo. Precisamente en ambos dendogramas se reconocen dos grandes conjuntos (1 y 2). En el primer gran grupo encontramos aquellos fragmentos donde la trama es vertical, el espaciamiento es cerrado, la cantidad de elementos de la trama vertical es simple y la morfología de los elementos de la trama vertical es plana/ cuadrangular. Por otra parte, en los fragmentos del otro gran conjunto (2) encontramos una trama horizontal en las estructuras, por ende, comparten aquellos atributos referidos a la misma. Además de esto, en este grupo se agrupan aquellas improntas que exhiben una morfología circular de los elementos de la trama vertical. A su vez, si realizamos un corte a un nivel mayor de similitud, este gran grupo se subdivide en función de la cantidad de elementos de la puntada (simple, doble $(2 \times 1)$, simple y doble, simple y múltiple), la cantidad de elementos de la trama horizontal (doble ( $2 \times 1)$, simple y doble) y el espaciamiento.

Al complementar esta interrelación de variables con el análisis cuantitativo acentuaremos aún más las diferencias entre estos dos conjuntos. Las medidas tomadas a los elementos de trama nos muestran la división que ya habíamos registrado entre la morfología plana/ cuadrangular y la forma circular de los elementos (Tabla 1) Además, son ligeras las diferencias que permitirán dar lugar a las divisiones internas entre los grupos. A partir de estos indicios, y de los antecedentes consultados en anteriores investigaciones de la provincia de Córdoba, podemos afirmar que las cestas que dejaron su marca en los fragmentos del grupo 1 fueron confeccionadas a partir de la técnica denominada coiled (Adovasio 1977), aduja o espiralado simple. Por otro lado, el otro gran grupo corresponde a las cestas confeccionadas mediante la técnica wrapped twining (Adovasio 1997), "falso espiralado" según Serrano (1945), encordado envuelto para Tarragó y Renard (2001) y acordelado envuelto para Pérez de Micou (2005). Como vemos en la Tabla $\mathrm{n}^{\circ} 2$, hemos colocado las técnicas cesteras identificadas junto a las variables más relevantes para definirlas, y parte del análisis cuantitativo se resumió en la Tabla $n^{\circ} 3$. A continuación, presentaremos con mayor detalle estas dos técnicas cesteras. 
Tabla 1. Resultados de las principales variables para el análisis de improntas.

Table 1. Results of the main variables for the analysis of imprints.



Primeros resultados: la cestería en espiral

Como mencionamos anteriormente, uno de los rasgos más evidente a simple vista en los fragmentos de la muestra es la morfología de los elementos utilizados en la cestería cuya impresión cubre los tiestos. Encontramos con dos clases muy diferentes, por un lado, una que correspondería a un elemento de forma circular cuya morfología tiende a ser angosta y larga, y otra plana que tiende a ser cuadrangular. Abordaremos en primer lugar aquellos fragmentos que evidencian una morfología plana en los elementos de la trama de las improntas.

Del total de fragmentos de la colección, 11 muestran los rasgos de cestas confeccionadas con elementos de morfología plana/cuadrangular. Al cruzar esta variable el resto de los atributos encontramos que dichos fragmentos 


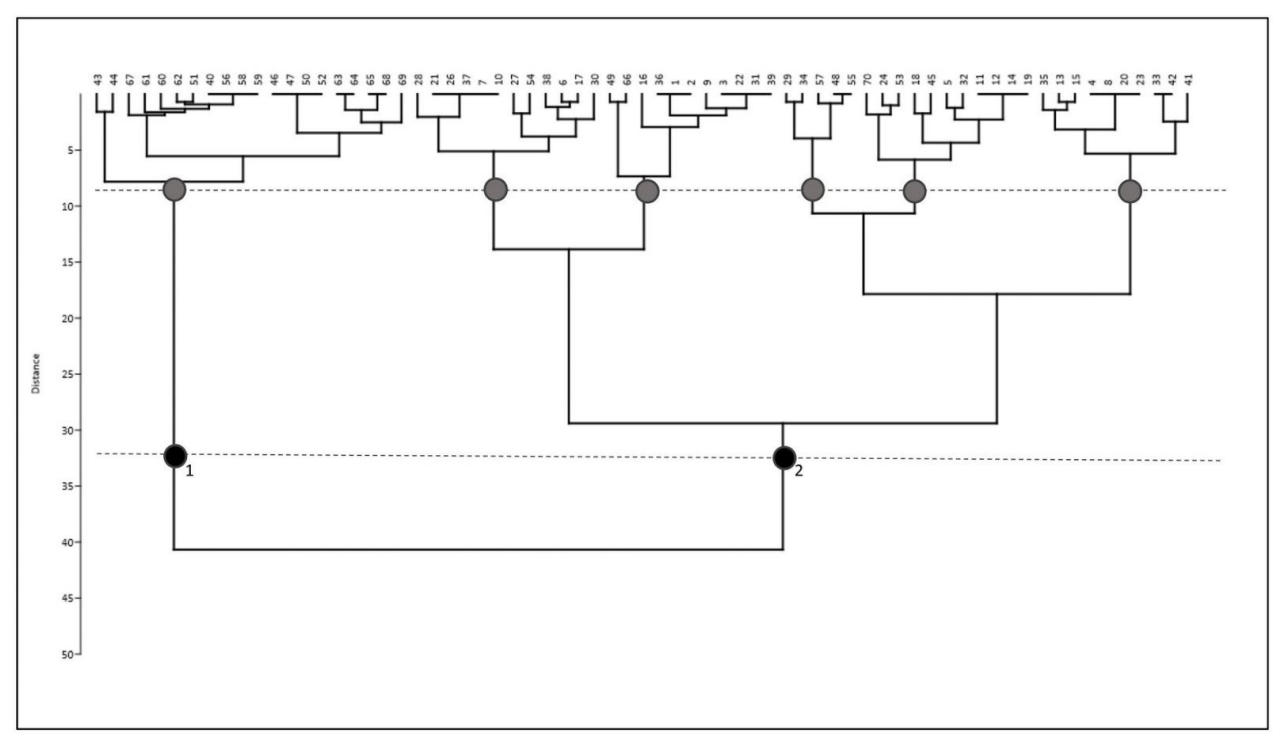

Figura 2. Dendograma aplicando el algoritmo de Ward con los dos grandes grupos definidos.

Figure 2. Dendogram applying Ward's algorithm with the two large groups defined.

poseen una trama vertical simple, cerrada y regular. Al poseer una trama cerrada, la urdimbre no puede observarse en los fragmentos. A partir de esto, definimos como técnica involucrada en la confección de las cestas a la denominada espiralado simple. En ella, los elementos pasivos (urdimbre) se disponen de forma horizontal desde la base hasta el borde de la pieza, mientras los elementos verticales activos (trama) van generando puntadas paralelas conformando la pieza. Dentro de esta clase, podemos definir la técnica, siguiendo a Adovasio (1977), como espiralado cerrado (close coiling), caracterizado por poseer puntadas simples una muy cerca de la otra.

El análisis cuantitativo mostró un mayor ancho en las puntadas de la trama de estos fragmentos en relación al resto de la muestra $(3,01 \mathrm{~mm}$ frente a la media de $1,73 \mathrm{~mm}$ para todo el conjunto). Sin embargo, pudimos encontrar diferencias dentro de este grupo que advierten que estamos ante dos tipos de interacciones que llevan a formas rítmicas particulares. Algunas estructuras textiles presentan elementos rectangulares con bordes poco marcados, que en ocasiones se tornan circulares (variedad I), mientras que otros exhiben formas rectangulares con bordes rectos bien definidos (variedad II). Las puntadas de ambos grupos atraviesan o rodean activamente la urdimbre sin dejar espacio entre ellas, por ende, pertenecen a la denominada puntada simple (simple stitch) (Adovasio 1977). Sin embargo, a pesar de pertenecer a la misma categoría, la puntada parece presentar ciertas diferencias. El espaciado entre las puntadas de la variedad II tiene un promedio de $3,77 \mathrm{~mm}$ mientras que para la variedad I es de $3,56 \mathrm{~mm}$. Si bien ningún fragmento de cesta se ha conservado y, por ende, no tenemos acceso directo a las puntadas para determinar el tipo, podemos suponer, a modo de hipótesis, ciertas interacciones a partir de la morfología que presentan los elementos de la estructura textil. De este modo, observamos que la variedad I, podría presentar una puntada denominada entrelazado (interlocking) o dividida (split), mientras que la variedad
Il podría corresponder a una puntada no entrelazada (non-interlocking) ${ }^{2}$. Si consideramos a la puntada como la unidad de análisis más pequeña, podemos encontrarnos con algunas diferencias en las formas de tejer dentro de la misma técnica. Sin embargo, al trabajar solo con impresiones de las estructuras textiles, no podemos avanzar en este aspecto.

\section{Otra técnica: encordado envuelto}

Los 48 fragmentos restantes corresponden al otro gran conjunto (2) definido en el dendograma. Para estos fragmentos, la trama mostraba indicios de elementos de morfología circular, a diferencia de los elementos de forma plana que aparecen en el espiralado simple. El promedio del ancho de la trama flexible en cada cruce es de 1,46mm, en contraposición con los 3,01 mm correspondientes para los elementos planos. La trama flexible para este conjunto es vertical abierta, paralela y diagonal, lo que permite visualizar claramente la trama semirrígida perpendicular a esta. A pesar de contar con diferentes cantidades de elementos, en casi todos los ejemplos de la trama horizontal prevalece un tipo particular de material que se infiere estar integrado de varillas semirígidas que son bien diferenciables entre sí. El ancho promedio de cada uno de estos elementos es alrededor de $1,12 \mathrm{~mm}$, donde el más pequeño es de $0,8 \mathrm{~mm}$ y el mayor de $2 \mathrm{~mm}$.

Los principales atributos de este conjunto son: la presencia de una trama doble (una horizontal semirrígida y otra vertical flexible), la morfología circular de los elementos que realizan las puntadas de manera alternada, y el entramado regular que se crea entre ambas tramas

2 En la puntada denominada entrelazado (interlocking) la fibra pasa diagonalmente a través de la parte superior de la puntada inmediatamente debajo, en la puntada dividida (split) uno de los elementos se bifurca para que otra puntada superior lo atraviese, y en la puntada no entrelazada (non-interlocking) la puntada se engancha a la urdimbre sin pasar por otro punto (Adovasio 1977: 62). Al no perdurar el material orgánico y sólo contar con la impresión de una cara de las caras de las piezas de cestería, estas interacciones son hipotéticas. 


\begin{tabular}{|c|c|c|c|c|c|c|c|}
\hline \multirow{3}{*}{\begin{tabular}{|c|} 
Tipo de Técnica \\
Variedad \\
Subvariedad
\end{tabular}} & \multicolumn{2}{|c|}{ Espiralado simple } & \multicolumn{5}{|c|}{ Encordado envuelto } \\
\hline & \multirow[t]{2}{*}{ I } & \multirow[t]{2}{*}{ II } & \multicolumn{2}{|c|}{ I } & \multicolumn{2}{|c|}{ II } & \multirow[t]{2}{*}{ III } \\
\hline & & & A & B & C & $\mathrm{D}$ & \\
\hline $\begin{array}{l}\text { Morfología (trama } \\
\text { flexible) }\end{array}$ & $\begin{array}{c}\text { Plana, } \\
\text { Rectangular }\end{array}$ & $\begin{array}{c}\text { Plana, } \\
\text { Cuadrangular }\end{array}$ & Circular & Circular & Circular & Circular & Circular \\
\hline Tipo de Trama & Vertical & Vertical & $\begin{array}{l}\text { Vertical y } \\
\text { Horizontal }\end{array}$ & $\begin{array}{l}\text { Vertical y } \\
\text { Horizontal }\end{array}$ & $\begin{array}{l}\text { Vertical y } \\
\text { Horizontal }\end{array}$ & $\begin{array}{l}\text { Vertical y } \\
\text { Horizontal }\end{array}$ & $\begin{array}{l}\text { Vertical y } \\
\text { Horizontal }\end{array}$ \\
\hline $\begin{array}{c}\text { Cantidad de } \\
\text { elementos (trama } \\
\text { flexible) }\end{array}$ & Simple & Simple & Simple & Simple & $\begin{array}{l}\text { Doble/ } \\
\text { Simple y } \\
\text { Doble }\end{array}$ & $\begin{array}{c}\text { Doble/Simple } \\
\text { y Doble }\end{array}$ & Simple/Múltiple \\
\hline $\begin{array}{l}\text { Espaciamiento } \\
\text { (trama flexible) }\end{array}$ & $\begin{array}{l}\text { Cerrado } \\
\text { regular }\end{array}$ & $\begin{array}{l}\text { Cerrado } \\
\text { regular }\end{array}$ & $\begin{array}{l}\text { Abierto } \\
\text { regular }\end{array}$ & $\begin{array}{l}\text { Abierto } \\
\text { regular }\end{array}$ & $\begin{array}{l}\text { Abierto } \\
\text { regular }\end{array}$ & $\begin{array}{l}\text { Abierto } \\
\text { regular }\end{array}$ & Abierto Irregular \\
\hline Tipo de urdimbre & Horizontal & Horizontal & Vertical & Vertical & Vertical & Vertical & Vertical \\
\hline $\begin{array}{l}\text { Posible Material } \\
\text { (urdimbre) }\end{array}$ & $\begin{array}{l}\text { Conjunto de } \\
\text { varillas }\end{array}$ & $\begin{array}{l}\text { Conjunto de } \\
\text { varillas }\end{array}$ & $\begin{array}{l}\text { Varilla } \\
\text { Rígida }\end{array}$ & Varilla Rígida & $\begin{array}{l}\text { Varilla } \\
\text { Rígida }\end{array}$ & Varilla Rígida & Varilla Rígida \\
\hline $\begin{array}{l}\text { Posible material } \\
\text { (trama horizontal) }\end{array}$ & & & $\begin{array}{c}\text { Varillas } \\
\text { semirrígidas }\end{array}$ & $\begin{array}{c}\text { Varillas } \\
\text { semirrigidas }\end{array}$ & $\begin{array}{c}\text { Varillas } \\
\text { semirrígidas }\end{array}$ & $\begin{array}{c}\text { Varillas } \\
\text { semirrigidas }\end{array}$ & $\begin{array}{c}\text { Varillas } \\
\text { semirrígidas }\end{array}$ \\
\hline $\begin{array}{c}\text { Cantidad de } \\
\text { elementos (trama } \\
\text { horizontal) }\end{array}$ & & & Doble & Doble/Simple & Doble & Doble/Simple & Doble/Simple \\
\hline Tipo de puntada & $\begin{array}{c}\text { Simple, } \\
\text { entrelazado }\end{array}$ & $\begin{array}{l}\text { Simple, No } \\
\text { entrelazado }\end{array}$ & & & & & \\
\hline
\end{tabular}

Tabla 2. Técnicas de tejido identificadas.

Table 2. Identified weaving techniques. (el espaciado entre los elementos de la puntada se mantiene medianamente regular a lo largo de todo el fragmento). Al igual que López Campeny (2011), en un primer momento identificamos estos tiestos como una variedad de espiralado, sin embargo, la información sobre improntas correspondientes a la región de Córdoba y los análisis realizados nos advirtieron que estamos en presencia de la técnica denominada encordado envuelto (Tarragó y Renard 2001). Este tipo de tejido se caracteriza por constituirse a partir de tres elementos: una urdimbre vertical, una trama semirrígida horizontal y una trama flexible. Este elemento flexible, que en este caso tiene morfología circular, es quien realiza la costura tejiendo entre ambos elementos. Las cestas construidas mediante esta técnica poseen patrones diferentes en sus caras (Adovasio 1977). Si tenemos en cuenta que las cestas fueron usadas como moldes, encontraremos en su interior (lado expuestos en los fragmentos) la trama horizontal intercalada por las puntadas de la trama vertical, mientras que, en el exterior de la cesta, se apreciaría la urdimbre vertical tomada por la trama flexible, esta vez, de manera horizontal y oblicua. Este lado exterior no se encuentra en ninguna de las improntas de los fragmentos de la colección. Tal como plantea López Campeny (2011), estas estructuras muestran una variante de la propuesta por Adovasio, ya que, en su cara interior, las puntadas se encuentran intercaladas y no alineadas. "Este efecto se logra debido a que las puntadas toman los elementos de la capa de urdimbres exterior (vertical) de manera doble, pero como pares alternos" (p. 244).

A partir de la variabilidad de este conjunto, hemos optado por dividirlo en tres variedades utilizando como criterio la cantidad de elementos de la puntada (elemento flexible), el espaciado de esta y la cantidad de elementos de la trama semirrígida. La variedad I (EE I) está conformada por aquellos tiestos que presentan un tramado abierto regular donde la trama flexible es simple, a su vez, esta variedad está dividida entre aquellos ejemplares que poseen una trama horizontal doble (subvariedad A), y otros con elementos dobles y simples de trama horizontal (subvariedad B) en sus estructuras. La segunda variedad (EE II) está conformada por aquellos fragmentos que poseen una puntada vertical doble $(2 \times 1)$ o simple y


Figura 3. Fragmentos arqueológicos que exhiben improntas de cestería en espiral.

Figure 3. Archaeological fragments that have coiled basketry imprints. 


\begin{tabular}{|l|c|c|c|c|c|}
\hline \multicolumn{1}{|c|}{ Tipo de } \\
Técnica & $\begin{array}{c}\text { Largo de } \\
\text { elementos de } \\
\text { trama vertical } \\
(\mathrm{cm})\end{array}$ & $\begin{array}{c}\text { Ancho de } \\
\text { elementos de } \\
\text { trama vertical } \\
(\mathbf{c m})\end{array}$ & $\begin{array}{c}\mathbf{N}^{\circ} \text { de } \\
\text { elementos de } \\
\text { trama vertical } \\
\text { por } \mathbf{c m}\end{array}$ & $\begin{array}{c}\text { Ancho de los } \\
\text { elementos de } \\
\text { la trama } \\
\text { horizontal }(\mathrm{cm})\end{array}$ & $\begin{array}{c}\text { Largo entre los } \\
\text { elementos de } \\
\text { trama vertical } \\
(\mathrm{cm})\end{array}$ \\
\hline $\begin{array}{l}\text { Espiralado } \\
\text { Simple }\end{array}$ & 0,37 & 0,3 & 3,33 & - & 0,09 \\
\hline $\begin{array}{l}\text { Encordado } \\
\text { Envuelto }\end{array}$ & 0,37 & 0,14 & 2,97 & 0,11 & 0,27 \\
\hline
\end{tabular}

Tabla 3. Promedios de las medidas del análisis cuantitativo agrupadas según las técnicas.

Table 3. Averages of the quantitative analysis measurements grouped according to the techniques.

doble. Esta también se subdivide según la cantidad de elementos de la trama horizontal, en dos subvariedades (C y D). Por último, contamos con dos fragmentos (variedad EE III) donde las puntadas de las tramas son diferentes, ya que alternan su orientación con respecto a la trama horizontal (paralelas o diagonales) con diferente cantidad de elementos de la puntada mostrando un espaciamiento abierto irregular. En estos fragmentos, las puntadas definen diversos diseños a partir del interjuego entre los elementos ya mencionados. La cantidad de elementos para la trama horizontal de esta variedad es simple o doble y simple (Tabla 2).

Cabe señalar que, si observamos las diferencias internas entre los tres grandes grupos del encordado envuelto, nos daremos cuenta de que estas son muy sutiles. Si bien tomamos como uno de los atributos diferenciadores a la cantidad de elementos de la puntada, vemos que para la variedad EE I la puntada es simple mientras que, para el resto, se da una simultaneidad de puntadas simples en conjunción con doble y múltiples. La diferencia existe, sin embargo, en términos de procedimientos técnicos y materiales estas disimilitudes son leves. Lo mismo sucede con la trama horizontal, donde la cantidad de elementos de esta es doble, alternando con unas pocas varillas simples, posiblemente semirrígidas, para la variedad EE I-B, variedad EE II -D y variedad EE III. Y es precisamente este último grupo el que marca mayor contraste con el resto al tener diseños que rompen con la regularidad de la muestra. Vale remarcar que este apartado no quiere minimizar la importancia de las diferentes elecciones realizadas por quien tejió la cesta, simplemente estamos marcando que las divisiones que encontramos dentro de la técnica encordado envuelto a partir de priorizar ciertos atributos son muy sutiles, por ende, la producción de estas cestas muestra cierta regularidad dentro de las diferencias. Si volvemos al dendograma (Figura $2)$, veremos que el segundo corte posee un alto nivel de similitud, acentuando las sutiles diferencias entre los agrupamientos. Si observamos el conjunto que corresponde al encordado envuelto encontraremos cinco agrupamientos similares a los presentados por el cuadro de la Tabla $n^{\circ} 2$, sin embargo, la variedad III no está discriminada en este análisis.

Como señalamos, la variedad III de la técnica de encordado envuelto es aquella donde las puntadas presentan distintos espaciamientos que se configuran como dibujos geométricos, tal como lo señaló Serrano (1955). Del mismo modo, en las investigaciones del Valle de Punilla efectuadas por Figueroa et al. (2011), los autores afirman: "en ocasiones pudimos identificar diseños decorativos: bandas paralelas y líneas en zigzag formando rombos" (p. 22). También López Campeny (2011) definió como tipo 5 (variedad c) a aquellos ejemplares con estructura simple donde las puntadas presentan una orientación diagonal y una trama abierta a intervalos irregulares. En nuestra muestra solo contamos con dos ejemplares que conforman la variedad III. Estos fragmentos muestran diseños que se crean al agrupar las puntadas en ciertas zonas de la cesta. Uno de los fragmentos exhibe puntadas en diagonal con una trama abierta y regular, alrededor de éstas, puntadas de pequeño largo con una "trama cerrada" delimitan el espacio. El otro fragmento, como vemos en la Figura $n^{\circ} 4$, presenta un diseño más regular donde las puntadas se encuentran agrupadas de a tres formando un diseño oblicuo. Esta franja está rodeada a ambos lados por puntadas simples en diagonal que
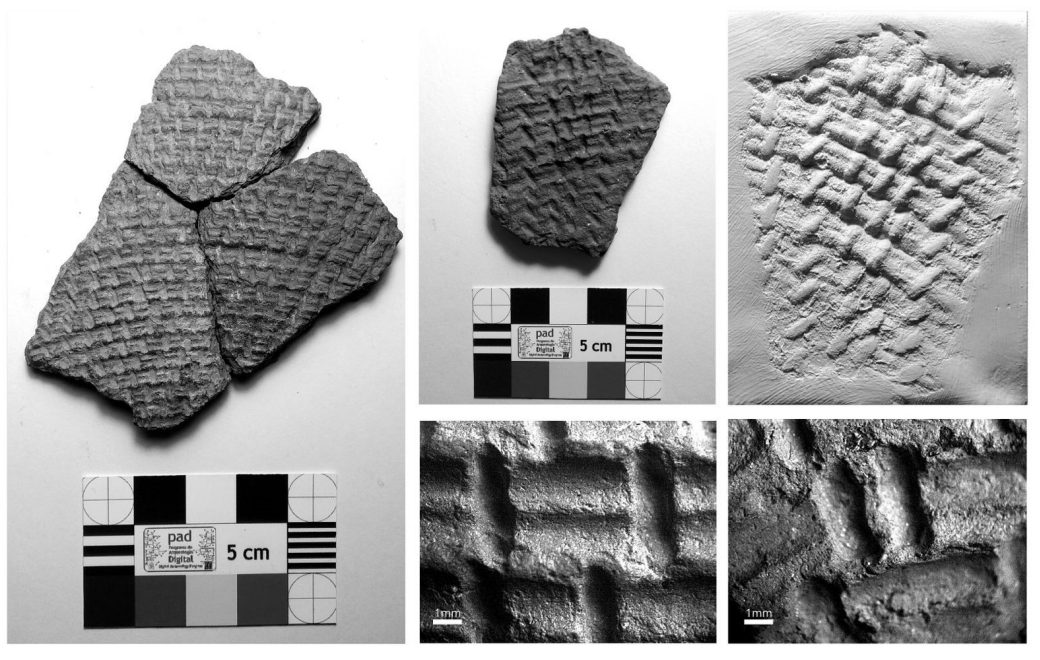

Figura 4. Fragmentos confeccionados a partir de un molde tejido a través de la técnica de encordado envuelto.

Figure 4. Fragments made from a mold woven through the wrapped twining technique. 

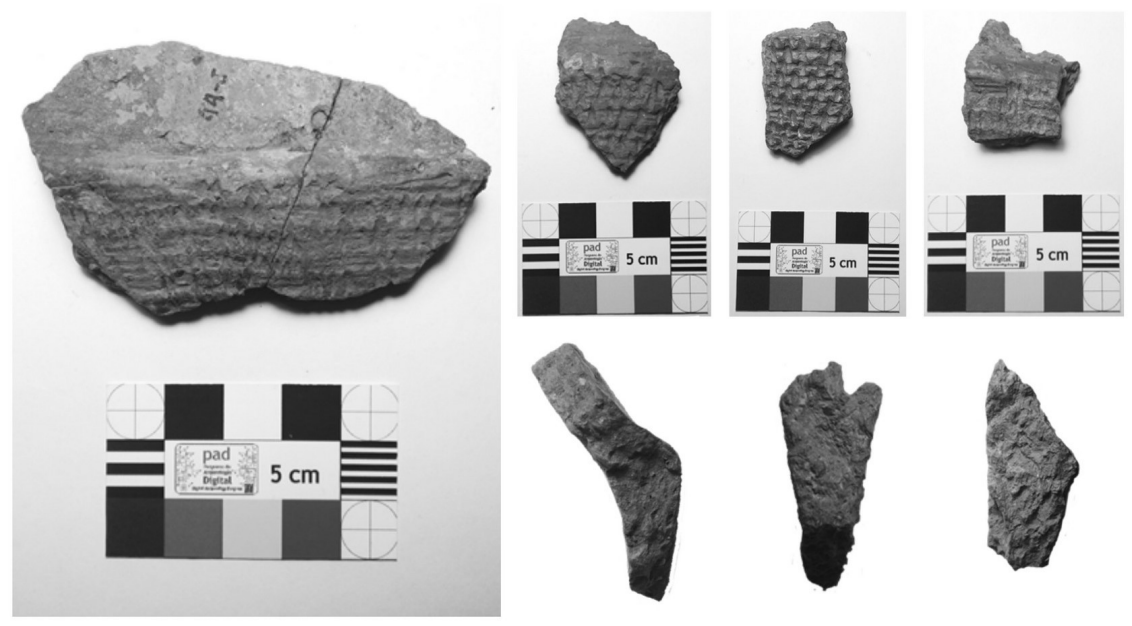

Figura 5. Puntos angulares donde se observa la unión entre las dos técnicas de manufactura.

Figure 5. Corner points where the union between the two manufacturing techniques is observed. acompañan el ángulo del sector central.

Dentro de la muestra, nos encontramos con 5 fragmentos cerámicos que exhiben la técnica de encordado envuelto en sus improntas y que corresponden a puntos angulares del cuerpo de las vasijas. Estos fragmentos presentan un contorno bien definido que genera un ángulo entrante que establece la división entre la parte inferior, donde se encontraba el molde, y la superior. Esto genera una curva discontinua en el perfil.

Al mismo tiempo, con respecto al grosor de estos fragmentos, encontramos que el ancho de las paredes en el punto de intersección es mayor $(12,6 \mathrm{~mm})$ que la parte inferior con impronta y la superior $\sin (6,5 \mathrm{~mm}$ y $6,3 \mathrm{~mm}$ respectivamente). A pesar de contar con estos fragmentos, su tamaño reducido no nos permite calcular el diámetro aproximado que tuvieron las canastas utilizadas como moldes. Por otro lado, para la técnica del espiralado simple tenemos un solo ejemplar de Villa de Soto (una urna que no pertenece a esta colección), donde la unión entre el molde de cesta y la parte superior presenta mayor continuidad, pudiendo llegar a determinarse como punto de inflexión. Este ejemplar, al igual que nuevas piezas obtenidas de nuestro trabajo de campo, serán analizadas en próximos acercamientos enfocando la producción cestera y cerámica como "tecnologías superpuestas" (López Campeny 2011).

Por último, uno de los fragmentos confeccionados mediante esta técnica presenta un nuevo elemento que no mencionamos hasta aquí. El fragmento 42/44-5, que pertenece a la variedad II $-C$, corresponde al de una base, que se eleva unos centímetros hacia las paredes (Figura 5). La impronta cubre la totalidad de la superficie externa salvo en la unión entre la base y las paredes. Para unir ambas partes del tejido se utilizó un hilo o cuerda cuya torsión es hacia la izquierda, en " $Z$ ", con un diámetro de $1 \mathrm{~mm}$. Este presenta una clara similitud con los hilos utilizados para la confección de redes cuya impronta encontramos en otros tiestos de la colección (los cuales serán analizados en detalle en próximos trabajos).
Considerando las posibles materias primas existentes hoy en la región, podemos pensar que este hilo podría haber sido confeccionado a partir de Bromelia urbanianum (caraguatá) ${ }^{3}$, especie que actualmente se encuentra en Soto formando "matas sobre las paredes de los cerros extendiéndose al piso de piedemonte" (Geisa y Martinez 2013:14). Ya Serrano (1945) señalaba la posible utilización de caraguatá para las redes, planta que según el autor se ubicaba en las barrancas altas de Cruz del Eje. De esta planta se extrae una fibra fina pero muy resistente que soporta bien el agua, de allí su utilización por diversos grupos para confeccionar redes de pesca y cordeles de arcos (Arenas 1992).

\section{Consideraciones finales}

A partir de la muestra pudimos describir y agrupar los fragmentos cerámicos para arribar a las técnicas utilizadas en la confección de las cestas que luego sirvieron de molde para la producción cerámica (Laguens 2017). A partir de la aplicación del código analítico descriptivo y la realización del análisis multivariado, pudimos diferenciar los fragmentos y llegar a agruparlos a partir de la interrelación de variables como la morfología de los elementos, cantidad de urdimbre y trama por cruce, espaciamiento, orientación, etc. Esto se complementó con un acercamiento cuantitativo que implicó medir las puntadas de la trama, los elementos y el espaciamiento de estos en las estructuras. Una vez identificadas las dos técnicas cesteras, espiralado simple para la menor cantidad de fragmentos y encordado envuelto en la mayoría, analizamos las diferencias dentro de las mismas.

Para los fragmentos identificados como espiralado simple, observamos ciertas diferencias a partir de la morfología de los elementos de la trama. Para discriminar entre estos nos centramos en uno de los momentos clave del hacer una cesta. Como señala James Adovasio (1977):

3 Los términos "chaguar" y "caraguatá" son formas de denominar a las bromeliáceas. En las áreas de influencia guaraní es común la utilización del término "caraguatá" mientras que el término "chaguar" es de origen quichua (Arenas 1992) 

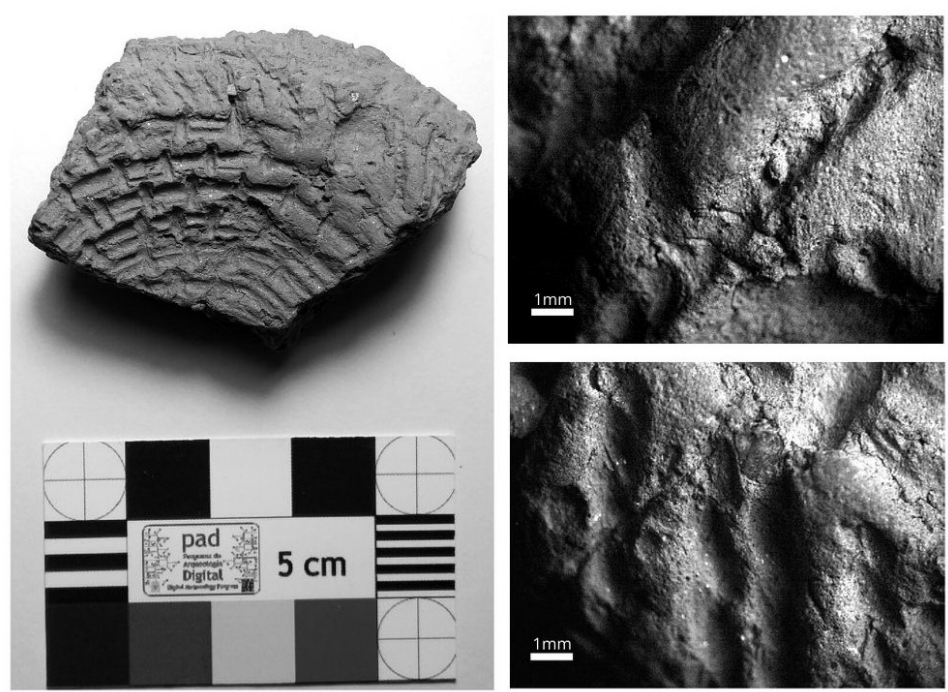

Figura 6. Fragmento 42/44-5 con improntas de la técnica de encordado envuelto.

Figure 6. Fragment 42 / 44-5 with imprints of the wrapped twining technique. uno de los problemas para identificar las interacciones entre la trama y la urdimbre en este tipo de cestos se halla en poder discriminar donde se genera la puntada y sus consecuencias. Es por ello que el nacimiento de la puntada constituye un momento clave del proceso de construcción entre la urdimbre, la trama y la persona que tejió. Vale remarcar que es la repetición rítmica de estos movimientos entre sí lo que da la apariencia regular final, cada puntada se levanta sobre la anterior y crea la energía para el movimiento siguiente (Ingold 2008). A pesar de las limitaciones de trabajar con las huellas de las estructuras, tratamos de discriminar las formas de realizar la puntada dentro de esta técnica, de esta manera, proponemos la utilización de una puntada entrelazada o dividida para la variedad I, y una posible puntada no entrelazada para la variedad II. Al mismo tiempo, si bien los elementos de la trama parecían ser similares, los fragmentos de la variedad II presentaban un mayor ancho y espaciado.

Por otro lado, si bien la urdimbre no es visible en nuestros fragmentos por corresponder a un espiralado cerrado, a partir de otras investigaciones podemos sugerir que las mismas podrían estar constituidas a partir de múltiples elementos formando un haz. Esto se atestigua en los calcos realizados en plastilina por Serrano (1945), a partir de algunos fragmentos de la colección Magnin procedentes de San Roque, Valle de Punilla. Por su parte, Bonofiglio y Herrera (1979) sugirieron la utilización de juncos, totoras y paja brava, esta última por su capacidad de realizar tramas pequeñas. En este sentido, es de gran aporte el trabajo de Pérez de Micou y Ancibor (1994) realizado sobre cestos confeccionados mediante la técnica de espiralado simple provenientes de Antofagasta de la Sierra, Catamarca. Las autoras demuestran la utilización de diferentes partes de una misma planta para los distintos elementos de la cesta. De este modo, la urdimbre es confeccionada a partir de un haz de pastos, situación similar a la hipotetizada para nuestro caso, mientras que las puntadas están realizadas por los nervios medios de la gramínea utilizada (Cortaderia). En la actual Villa de
Soto, esta se encuentra formando matas en diferentes puntos del paisaje por lo que podría haber sido la materia prima de las cestas. Sin embargo, aún no hemos podido sugerir que especies podrían haber estado involucradas en aquellas cestas de técnica de encordado envuelto, pues tanto sus puntadas, como las varillas semirrígidas que componen la trama horizontal, son diferentes. Como vemos, cada técnica podría requerir materiales diferentes para su construcción, en ese caso, cada "hacer" una cesta es distinto. Estamos frente a ritmos particulares según el tipo de tejido que se realice.

En cierto sentido, se vuelve interesante pensar en las variantes morfológicas locales de las diferentes cestas confeccionadas. Para poder contextualizar las diferencias es necesario ahondar en las múltiples articulaciones que poseen las cestas, los materiales, los humanos y nohumanos. Para esto es necesario tener en cuenta que dicho proceso es mucho más que una serie de pasos técnicos con miras a un objetivo. Hacer un cesto exige más que la interrelación con materiales locales, implica la superposición de diversos ritmos sociales, creativos, técnicos y cíclicos (naturales) donde el cesto no es el fin, es parte del devenir (Ingold 2008). Una potente vía de análisis a desarrollar consistiría en abordar la cesta como un ensamble (Jones y Alberti 2013), comprendiendo cómo los elementos se conjugan para dar lugar a una forma particular de cesta. En este acercamiento, la noción de ensamble reemplaza a la de contexto, haciendo referencia a "la disposición, organización y ajuste mutuo de componentes heterogéneos" (Jones y Alberti 2013: 29). La cesta emerge de un ensamble y es un ensamble que forma parte de diversas articulaciones al ingresar en otros procesos; articulaciones entendidas como "configuraciones variadas vislumbradas a través del rastreo de las conexiones de personas y materiales en procesos tecnológicos pasados" (Conneller 2011:4). De este modo, el hacer una cesta implica articulaciones que van más allá del ritmo del hacer propiamente dicho, las que entrelazan diversos elementos, tales como 
los ciclos estacionales de plantas, sus relaciones con el río, la localización de la arcilla y todas las múltiples relaciones entre humanos y no humanos. Al considerar el proceso de construcción de una cesta definimos ciertas articulaciones que estarán presentes en la interactividad: la relación entre las materias primas, su preparación diferencial, el proceso mismo del tejer donde entran en juego las técnicas (como las descriptas arriba), la memoria, las habilidades, las tradiciones, el saber cómo, etc. A su vez, la propia noción de ensamble nos permite movernos en diferentes entramados donde la cesta es un elemento más del conjunto. Por ejemplo, si tomamos a la cesta como contenedora de alimentos, veremos cómo ingresa en un nuevo entramado. Si la vemos como contenedora de un recurso de recolección local como es la algarroba, ingresamos en nuevas articulaciones con otros ritmos cíclicos implicados (meses de diciembre, enero y febrero) donde el algarrobo da sus frutos (Laguens 1999a, González Navarro 2010, Serrano 1945). Además, diversos registros señalan que esta recolección nucleaba anualmente a las personas de diferentes asentamientos (Laguens 1999) (hecho que produjo varios enfrentamientos entre los encomenderos por la disputa de la mano de obra indígena) (Piana 1992). En este sentido, la cesta ingresa en un ensamble que requiere, para su comprensión, una escala mayor que contemple la región teniendo en cuenta las diversas trayectorias de grupos movilizándose hacia los algarrobales, pues, como señala González Navarro (2010), estos sitios constituían importantes lugares para el intercambio entre poblados diferentes, y en términos de movilidad, constituían itinerarios transitados regularmente pues al igual que el algarrobo, los frutos del chañar y molle también se encontraban en zonas específicas de los valles (Laguens y Bonin 1987). Como vemos, esta es una de las ventajas de analizar el devenir a partir de este enfoque, ya que contamos con la posibilidad de movernos en diferentes escalas y al mismo tiempo considerar como los ensambles están formados a partir de otros ensambles (Harris 2017). En ellos, las cestas, sus formas de fabricación, su uso, su imbricación con las vasijas, y las tramas de relaciones en que todas ellas participaron, resaltan desde el momento del mínimo gesto técnico de la generación de la puntada y las implicancias entre tramas y urdimbres. De esta manera, vemos como las cestas estuvieron activamente ensambladas en el cotidiano devenir de estas sociedades.

Córdoba Capital, 6 de agosto de 2020

\section{Agradecimientos}

A la Secretaria de Ciencia y Tecnología de la Universidad Nacional de Córdoba por financiar el proyecto en curso. Al Dr. Andrés Laguens por las sugerencias y la lectura atenta. A los miembros del proyecto "Re-ensamblando la Arqueología del Chihimi Sei", especialmente a los miembros del Museo Comunitario de Villa de Soto. Al Museo de Antropología (UNC) y al Instituto de
Antropología de Córdoba (IDACOR) por el apoyo brindado. A Paola Franco por la confección del mapa, y a Veronica Mors por su ayuda.

\section{Bibliografía}

Adovasio, J. M. (1977). Basketry technology: a guide to identification and analysis. Manuals on Archaeology, Aldine Publishing Company.

Arenas, P. (1992). El Chaco, su gente y las plantas. Texto del catálogo de exposición: Etnobotánica 92. Edición de la Universidad de Córdoba, España.

Argüello de Dorsch, E. A. (1983). Investigaciones arqueológicas en el Departamento Punilla (Provincia de Córdoba, Rep. Argentina). Sitio: C. Pun. 39. En Comechingonia, vol. 1, 41-60.

Bonofiglio, M.; Herrera, M. (1979). Impresiones de cestería en la cerámica de Río Segundo, Córdoba. Museo arqueológico Aníbal Montes, Publicación n5.

Calo, C. (2008). Improntas del pasado: las canastas de Cardonal. Revista Española de Antropología Americana, vol. 38, n², 39-55.

Dantas, M.; Figueroa, G. (2008). ¿Uniformidad o variabilidad tecnológica y estilística en la alfarería del sector centro-oeste de las Sierras Centrales (Córdoba Argentina)? ArqueoWeb, vol. 10, n². URL: http://www. ucm.es/info/arqueoweb/

Emery, I. (1966) The Primary Structures of Fabrics. The Textile Museum, Washington D.C.

Fabra, M.; Laguens, A. (1999). Análisis tecnológico de improntas de cestería en fragmentos cerámicos de Córdoba, Argentina. Actas del XII Congreso Nacional de Arqueología Argentina, La Plata, Tomo II, 25-34.

Figueroa, G.; E. Pautassi y M. Dantas. (2011). Técnicas cesteras y cerámica arqueológica de las Sierras Centrales de Córdoba, República Argentina. ArqueoWeb, vol. 13, 15-31. URL: https://webs.ucm.es/info/arqueoweb/pdf/13/ figueroapautassidantas.pdf

Gardner, G. A. (1919). El uso de tejidos en la fabricación de la alfarería prehispánica en la Provincia de Córdoba. Revista del Museo de La Plata, XXIV, Segunda Parte, 128-163.

Geisa M.; Martinez E. (2013). Proyecto Área Natural Protegida en el río de Villa de Soto. Municipalidad de Villa de Soto, Córdoba.

González Navarro, C. (2012). Una Aproximación Al Territorio Indígena Prehispánico. Córdoba (Siglo XVI). Andes, vol. 23. URL: https://www.redalyc.org/articulo. 
oa?id=127/12726101002

González, A. R. (1943). Arqueología del Yacimiento Indígena de Villa Rumipal. Publicaciones del Instituto de Arqueología, Lingüística y Folklore IV. Universidad Nacional de Córdoba.

González, A. R. (1943). Paradero indígena de Soto (Córdoba). Anales del Museo Argentino de Ciencias naturales, Tomo XLI, 53-70.

Hammer, $\varnothing$; Harper, D. A. T; Ryan, P. D. (2001). Paleontological Statistics software package for education and data analysis. Palaeontologia Electronica 4 (1).

Harris, O. (2017). Assemblages and scale in Archaeology. Cambridge Archaeological Journal, vol.27, n¹, 127-139.

Ingold T. (2008). The perception of the environment Essays in Livelihood, Dwelling and Skill. Routledge. Jones A.; Alberti. B (2013). Archaeology after interpretation. En Archaeology after interpretation: returning materials to Archaeological Theory. (Left Coast Press, cap. 1, pp. 1535). California: B. Alberti, A. M. Jones, y J. Polland (Eds.).

Laguens, A. y Bonnin, M. (1987). Espacio, paisaje y recursos. Estrategias indígenas alternativas y complementarias en la cuenca del río Copacabana (Dto. Ischilín, Córdoba, Arg.). Sitio El Ranchito: 1000 a.C.-1600 d.C. Publicaciones del Instituto de Antropología, Tomo XLV, 159- 204.

Laguens, A. (1999). La recolección de algarrobo en la economía indígena del Norte de Córdoba. En: En los tres reinos. La recolección en el Cono Sur. (Ed. Magna, Tucumán, cap. 14, pp.187- 196) Tucumán: A. Kornstaje y C. Aschero (Eds.).

Laguens, A. (2017). Mimesis, citas y copias. Reflexiones sobre los modos de hacer y ser en Soto, Córdoba, en tiempo precoloniales. Trabajo presentado en las XII Jornadas de Investigadores en Arqueología y Etnohistoria del Centro Oeste del País, Río Cuarto, Universidad Nacional de Río Cuarto.

Laguens, A. y Fabra, M. (1999). Análisis tecnológico de improntas de cestería en fragmentos cerámicos de Córdoba, Argentina. Actas del XII Congreso Nacional de Arqueología Argentina, La Plata. Tomo II, 25-34.

Laguens, A; Bonnin, M.; Abalos Luna, M.; Cruz, C.; Fernández, M.; Ferreira M.; Freites, N.; Laguens, G.; Ochoa, S.; Pesci, A. y Quintero, M. C. (2017). Ritmos, tiempos y duraciones en la vida cotidiana de las sociedades agroalfareras de la región de Villa de Soto, Córdoba, Argentina. Trabajo presentado en las XII Jornadas de Investigadores en Arqueología y Etnohistoria del Centro Oeste del País, Río Cuarto, Universidad Nacional de Río Cuarto.
Lahitte, H. y Cascardi, J. (1980). El Análisis Descriptivo: su funcionamiento. Cuadernos LARDA. Año 2, n4 La Plata: Facultad de Ciencias Naturales y Museo.

Lefebvre, H. (2004). Rhythmanalysis. Space, time and everyday life. Editorial Continuum.

López Campeny, S. (2011). La impresión es lo que cuenta... Análisis de improntas textiles. Casos arqueológicos para Santiago del Estero. Relaciones de la Sociedad Argentina de Antropología, vol. 36, 221-247.

Marcellino, A. J.; Berberían, E. E.; Pérez, J. A. (1967). El yacimiento arqueológico de Los Molinos (Dpto. Calamuchita- Córdoba). Publicaciones del Instituto de Antropología, Tomo XXVI, Córdoba.

Mlekuz, D. (2014). Exploring the topography of movement. En Computational Approaches to the Study of Movement in Archaeology. Theory, Practice and Interpretation of Factors and Effects of Long Term Landscape Formation and Transformation (De Gruyter, cap. 1, pp. 5-22). Berlin: S. Polla y P. Verhagen (Eds.).

Outes, F. (1911). Los tiempos prehistóricos y protohistóricos de la provincia de Córdoba. Revista del Museo de La Plata, Tomo XVII (seg. serie, IV), 261-374.

Pérez de Micou, C. (2000). Dos cestas decoradas en el ajuar de la momia $N^{\circ} 136$ de Loro Huasi (Catamarca, República Argentina). Actas de la XIII Reunión Anual del Comité Nacional de Conservación Textil 1999. Arica, Chile, pp. 103- 110.

Pérez de Micou, C. (2001). Cestería y Cordelería para los muertos. Revista Chungará, vol. 33, n¹, 137-144.

Pérez de Micou, C. (2005). Pautas descriptivas para el análisis de cestería arqueológica. En Tejiendo sueños en el Cono Sur. Textiles Andinos: Pasado, Presente y Futuro. Grups d'Estudis Precolombins, pp. 27-35). Barcelona: V. Solanilla Demestre (Ed.).

Pérez de Micou, C.; Ancíbor, E. (1994). Manufactura cestera en sitios arqueológicos de Antofagasta de la Sierra, Catamarca. Journal de la Societé des Américanistes, vol. 80, 207- 216.

Piana, R. 1992. Los indígenas de Córdoba bajo el régimen colonial 1570-1620, Ediciones del Autor, Córdoba, Argentina.

Scattolin, M. C., Bugliani, M., Cortés, L., Calo M., Pereyra Domingorena, L., y Izeta, A. (2009). Pequeños mundos: hábitat, maneras de hacer y afinidades en aldeas del valle del Cajón, Catamarca. Relaciones de la Sociedad Argentina de Antropología, vol. 34, 251-274. 
Serrano, A. (1945). Los Comechingones. Serie Aborígenes Argentinos I. Publicación del Instituto de Arqueología Lingüística y Folklore Dr. Pablo Cabrera. Imprenta de la Universidad Nacional de Córdoba, Córdoba, Argentina.

Shennan, S. (1992). Arqueología cuantitativa. Editorial Crítica, Barcelona.

Tarragó, M. y Renard, S. F. (2001). Cerámica y cestería arqueológica del Valle de Yocavil. Una aproximación a partir de improntas. Actas del XIII Congreso Nacional de Arqueología Argentina, Tomo I, 513-528. Universidad Nacional de Córdoba, Córdoba, Argentina.

Tell, S, y Castro Olañeta, I. (2011). El registro y la historia de los pueblos de indios de Córdoba entre los siglos XVI y XIX. Museo de Antropología de la UNC. Revista del Museo de Antropología, n4, 235-248. 